\title{
Quantum Physics and Reality
}

\author{
Rudolf Haag \\ D-83727 Schliersee-Neuhaus, Waldschmidtstr. $4^{\text {b }}$ \\ Reprint requests to Prof. R. H.
}

Z. Naturforsch. 56a, 76-82 (2001); received January 12, 2001

Presented at the 3rd Workshop on Mysteries, Puzzles and Paradoxes in Quantum Mechanics, Gargnano, Italy, September 17-23, 2000

\begin{abstract}
Apparent paradoxes in Quantum Physics demand a sharp distinction between a "real part of the world" and the realm of possibilities. In the former we may distinguish individual elements (coarse events) relating to space-time, with causal connections between them respecting the relativistic locality principle. The latter, quantitatively described by probability assignments conditioned on existing facts and depending on the definition of equivalence classes of situations, cannot be subdivided in space-time categories. There are global correlations (Pauli principle...). The cut between the realms of facts and possibilities implies an evolutionary picture of the "real world" in which the (generalized) arrow of time assumes basic significance. Some deficiencies of existing theory are pointed out.
\end{abstract}

Key words: Quantum Philosophy; Entanglement; Irreversibility.

\section{Introduction}

In the title of this conference there appear the words mysteries, puzzles, paradoxes. Mysteries may be unfathonable, puzzles can be solved and paradoxes may either point out a puzzle or illustrate a mystery. The most essential issue in the debates between Einstein and Bohr concerned the meaning of reality. This discussion has not subsided as illustrated by titles like "On the search for reality", "Reality or Illusion?". Long before the advent of Quantum Theory it has been a battle ground for philosophers. The naive person instinctively feels that he is confronted by an external world on which he has little influence. On the other hand, the only knowledge I can have of this world resides in my mind: it is the contents of my consciousness. Thus arose the so called mind-body problem in philosophy. What is the relation of the inner world of my consciousness and the assumed outer world? Extreme idealists tried to reduce everything to consciousness and eliminate the notion of an outside world. Extreme materialists tried to explain consciousness as synonymous with states of the brain. Others, e.g. Popper proposed a dual model of two interacting worlds.

For most physicists such questions appeared entirely irrelevant for their work. They proceeded to classify recurring phenomena, study their relations, aiming at a description of the outside world and the discovery of laws of nature. The basic notions entering in this de- scription were matter, fields and their ordering in space and time. The description of matter was reduced to a few elementary building blocks, say electrons and nuclei: fields were described as attributes of spacetime points. Space and time, considered together as space-time, was described as a 4-dimensional continuum with known geometry. The time development was given by strictly deterministic laws: given full knowledge of the configuration of matter and fields at one time, the configuration at any other time was uniquely determined. The success of this endeavour was spectacular. Its applications reached far beyond the consciousness of scientists. It changed the way of life of mankind.

But it also became evident that some basic tenets of this approach could not be upheld. There was some element of discreteness in nature, a discontinuity, manifested in the stability of matter, quantum jumps or, more generally in processes defying a detailed description in space-time which had to be regarded as indivisible units, unpredictable events. The standard language of Quantum Theory, emerging from the mathematical formalisms of Matrix Mechanics and Wave Mechanics after agonizing discussions, puts the observer in a central position. The theory makes statements about the appearance of nature and refrains from ontological speculations about nature as such.

John Archibald Wheeler liked to express this in a paradox: "No phenomenon is a phenomenon unless it is ob- 
served." But who or what qualifies as an observer? One answer could be that it is the faculty of consciousness. This position has been taken by several eminent physicists, most sharply stated by Eugene Wigner 1963: "If one formulates the laws of Quantum Mechanics in terms of probabilities of impressions, these are - ipso facto - the primary concepts with which one deals". When I talked with John Wheeler about the meaning of his "paradox" he ultimately agreed: "It has nothing to do with the mind".

\section{Objective Appearance. Individual Phenomena and Equivalence Classes}

But this is not entirely a matter of opinions. It can be clarified considerably and this is not only of academic interests; it may have consequences for the development of the theory. In the passage from individual consciousness to statements about nature there are several steps. The first one, essential for engaging in physics at all, is the conviction that there are other human beings with their own minds and that I can commnicate with them about impressions. In this process we find a remarkable agreement, a one to one correspondence between certain of our impressions (not all impressions) and this leads to the notion of objective phenomena. These constitute the raw material from which physics starts. The impressions of an individual are unreliable. It is the agreement between impressions of many which is needed. We instinctively look for a cause for such agreement and attribute it to the existence of an external world. This is indeed the simplest mental picture of the state of affairs but it is of no deep consequence whether we speak of an objective phenomenon or of the perception of a fact in the external world. Still, for the description of objective phenomena, the realistic language is much more convenient. The experience of separability of complex appearances into individual phenomena leads to the notions of space and time. Thus the prototype of such a description of a basic phenomenon is the assignment of a region in space and time to a conspicuous appearance. If the appearance changes continuously with increasing time we speak of an object, if there is an abrupt change we speak of an event.

All ultimate results of measurements are described by such an assignment of a space-time region to an objective phenomenon (position of a pointer of an instrument, emergence of a dot in a photographic emulsion, click of a detector ...).
In the systematic study of relations between objective phenomena a next step is essential. We recognize similar situations and form equivalence classes (different times, different space regions, different individual objects). The individual case is replaced by an ensemble of many apparently equivalent situations. I used the world ensemble purposely in this context without any connection with probability. It is an absolutely essential step in physics, whether classical or quantum, to define equivalence classes of phenomena, to focus on equivalence classes of processes and use inductive inferences to come back from the ensemble to statements about an individual case. And it is a highly non trivial step. What are equivalent regions in space-time? What equivalent objects? The possibility of an answer is due to an extraordinary kindness of nature and involves a considerable amount of physical experience. We need that the laws are (roughly) invariant under translations in space and time, and we find that they are also invariant under rotations and Lorentz transformations. Taken together, these define a symmetry group of space-time, the Poincare group. This allows us to establish a common reference frame in space-time in which the observers concerned can order their impressions about basic phenomena. Two space-time regions are equivalent if one results from the other by a symmetry operation. Let us not pursue here the much more difficult question of how we recognize equivalent phenomena. Having succeeded in forming representative ensembles, having achieved the reproducibility of experiments and relying on inductive inferences we can extend the realm of objective appearance enormously beyond the basic phenomena. We may say, for instance that we sent a laser beam through a reaction chamber, basing this confidence on many preceeding monitoring experiments. Or we may say that the star Sirius existed for many millions of years though no human being could have observed this. Some cosmologists would be willing to extend objective appearance to the scenario of the big bang.

To repeat: physics is based on the existence of a realm of objective appearance. Objective means that this is not the content of consciousness of an individual but the agreement between impressions of many. The second pillar, the recognition of equivalent situations, leading to reproducible experiments, allows us to extend this realm from directly observed objective phenomena to statements gained with high confidence level by stringent reasoning based on many observations and inductive inference. We need not interpret this realm as representing (the perception of) features of an outside 
world but we may do so without fearing contradictions, and indeed this is the simplest rationalization of a cause for the agreement and it provides the most convenient language. So we shall do so and call extended objective appearance just "reality" and its elements "facts". Still we must remember that the whole building ultimately rests on impressions of consciousness (Wigner) though not on individual consciousness but on agreement of many as to what constitutes a fact.

\section{Theory vs. Ontological Models}

But physics does not stop at describing objective phenomena and results of experiments. It tries to unify the understanding by the invention of a theory. When I used the word invention I followed the authority of Einstein who stressed that, at least at an advanced level, the theory is not deduced from experiment but has to be freely invented. In this invention we may use, and often have to use, concepts and mathematical structures which are rather indirectly related to phenomena. In classical theory already the idea of fields pervading space-time is such a notion. Still, before the advent of Quantum Theory it was believed that the theory could be regarded as an extrapolation from the realm of objective appearance and could ultimately yield an ontological model of the universe.

In Quantum Physics this hope is barred by several features. First, there appears to be an intrinsic indeterminacy which forces us to consider besides the realm of objective appearance a realm of possibilities, potentialities. It is quantitatively described by probability assignments to which a large part of the mathematical structure of Quantum Physics refers. Probabilities for what? For the realization of an objective phenomenon, something which, with high confidence level, can be called a fact.

But parallel to this there is another problem. We like to explain the complexity of appearances in terms of simple constituent parts which can be classified. This is called reductionism and it has been an indispensable method in exact natural science because any symbol we use refers to some specific element or concept. The formalism of Quantum Theory suggests that most (if not all) of the ways by which we try to subdivide the universe must be regarded as approximations whose worth depends on particular circumstances. This applies most strongly to the division into individual objects. Somewhat better is the division into individual events but it is not precisely defined in existing theory. The best division we have, the one which constitutes the rock on which present theory is built, is the division of space and time. Let us note in passing that even this last rock is wavering if we strive for a synthesis of Quantum Physics with General Relatively. This is responsible for the great conceptual difficulties in the development of a Quantum Theory of Gravitation.

Let us begin with the notion of "object" or, more generally "physical system". Here we are faced with the famous topic of entanglement. In the simplest case, the entanglement of two particles originating from a common source, the violation of Bell's inequately means that it is impossible to attribute any notion of "objective state" to either one of the particles. The "two particle system" cannot be regarded as two systems of single particles. But entanglement reaches much further. According to the Pauli principle all electrons in the world are entangled. The spectacular effect of that - or rather its bosonic counterpart - is demonstrated in the experiments of Hanbury Brown amd Twiss. It refers to intensity correlations in the counting rate of coincidences from two detectors in which the coincidence signal is attributed to two photons emitted in entirely independent processes form two far separated regions of a star.

It is often said that entanglement shows the extreme non-local character of Quantum Physics. But this ignores the fact that there exists a well established, well tested, principle of locality which plays a crucial role in relativistic Quantum Physics. I have described the general frame of this synthesis of the basic principles of Quantum Physics with those of Special Relativity in my book "Local Quantum Physics" [1] (see also [2], [3]). There also many qualitative consequences are described. The best specific model within this frame existing to-day is the standard model of electroweak and strong interactions. Certainly this general frame and - a forteriori - the specific standard model do not represent ultimate wisdom. But such doubts and questions have no bearing on the analysis of the experiments mentioned above.

\section{Alice and Bob}

We have to distinguish between non-local features referring to probability assignments (for the realization of facts) wich are summarized in the notion of "quantum state" and the emergence of new facts which, in our context, are the clicks of detectors (events). The latter are, 
first of all, reasonably well localized in space and time and are represented in the theory by "local observables" associated with some specific space-time region. The former describe correlations which, in the example of the EPR-type measurements by Alice and Bob, are found in the joint probability distribution for the coincidence of clicks in the two stations. These depend on the choice of the experiments by both Alice and Bob and are of a nature which cannot be explained within the picture of two real particles travelling through space, each being in some state. One cannot explain the correlations of the events in terms of correlations of such assumed states (as in the classical example referring to Bertlsmann's socks). The clicks are primary objective phenomena, the particles are not. The locality principle says that whatever measurement Alice chooses to make, her counting rate is not influenced by anything Bob decides to do and furthermore that no superluminal transmission of effects or information enters in the analysis of the experiments.

Let us go carefully through the analysis of what Alice and Bob have to know, how and when they acquire this knowledge and why and when some "collapse of the wave function" occurs. First of all, the correlations refer to the statistics over many individual cases. One individual case ends with a pair of clicks, one in Alice's lab, the other in Bob's lab. Not arbitrary clicks but a pair belonging together because of a common cause which, in our simple example, is the decay process from which the two particles result. In the case of the Hanbury Brown and Twiss effect it is more subtle. There the common cause is described by the relevant quantum state resulting from the Bose statistics of two photons which, though emitted by independent sources, overlap at some time in the surroundings of the detectors. In any case Alice and Bob, in comparing their data, have to know which clicks belong together and for this purpose they have to record the time of each click (besides, of course, the orientation of the Stern Gerlach magnet and the signal received). For the evaluation of the statistics Alice and Bob can meet leasurely over a cup of coffee after the run of the experiment is over, each bringing their own data. "Coincidence" of a pair of clicks means that they result from the same (individual) decay. Thus, if Alice is closer to the source than Bob then it means a delayed coincidence on the side of Bob by the difference $\Delta T$ in flight time. If the distance from the source and the mean velocity of the decay products are known then $\Delta T$ is known. But this is not even necessary. They can treat $\Delta T$ as an unknown constant. Only for the cor- rect choice of $\Delta T$ in the analysis will they find the optimal correlation.

In the theoretical prediction the relevant quantum state is guessed from the preceeding objective appearance. In relativistic physics, but also if observations at different times are involved, it is better to use the Heisenberg picture. There the time of observation is included in the description of the "observable" while the state does not change with time as long as it remains undisturbed by interaction with other systems. But any emergence of a new fact changes the quantum state since the probability assignment for subsequent events is conditioned on the set of facts which are used in defining the ensemble of equivalent situations for which the statistics is evaluated. In our example we are not interested in the fate of the system after the occurrence of the clicks. So we need not consider the overall change of the quantum state. But if Alice and Bob decide to analyze the statistics asymmetrically, classifying the data by first taking Bob's results into account, then Alice has to consider the conditional probabilities resulting from this information. This means that she must subdivide her data into subensembles corresponding to the different results of the partner clicks on Bob's side. The "partial state" referring to Alice's lab is changed due to information received (much later) by looking at Bob's data. In this case, the "collapse of the wave function" (for Alice) is only a book keeping device which could have been chosen the other way around. The actual time of the occurrence of the clicks (apart from the identification of partners) is irrelevant.

To sum up: In the existing relativistic quantum theory there appear two distinct categories of concepts. On the one side there are "local observables" (typically representing detectors) whose response characterizes an event i.e. the emergence of an individual fact to which a rather well defined region of space-time can be assigned and which can be clearly distinguished thereby from other individual events in the universe. The algebraic relations of such observables ensure that causal connections between events conform with the causal structure of Minkowski space. On the other side there are probability assignments for possible events, summarized in the "quantum state". These are in general not factorizable with respect to a division of space-time. One may restrict attention to the probabilities of events in some region: this leads to the notion of "partial state" for the respective region. But the partial states of two disjoint regions do not determine the partial state in the union of the two regions. There may be correlations, 
even if the regions are far space-like apart. The origin of these non-local or holistic aspects is due to several features. One of them - existing also in classical mechanics - is the following. Several particles emanating from one event will carry the correlations imposed between them in the production process to different regions of space at later time. If these correlations refer to constants of motion they will remain noticeable much later at large distances. But the seemingly paradoxical features of the correlations in Quantum Physics tell us that we cannot attach any reality to individual particles. This message is conveyed already by the indistinguishability of the particles of one species, leading to Bose- or Fermi-statistics and thus to global entanglement. If we talk of particles in a specific process then these are part of the description of the relevant partial quantum state and thus refer to the probability for the occurrence of subsequent facts within an ensemble which in turn is defined by a judicious choice of an equivalence class of situations. It is, of course, tempting to assign some measure of reality to a quantum state, dissociating it from the choice of an ensemble. Thus it has been suggested to regard probabilities as intrinsic properties in an individual case, called "propensity" by Popper and "objective probability" by Mermin. One problem here is that, unless one talks about the state of the universe, the state refers to a "physical system" whose entanglement with other parts must be disregarded. We must also take into account that the probabilities are conditioned on some parts of objective appearance defining an equivalence class of processes.

\section{The Shadow of Schrödinger's Cat. Criticism of the Frame of Present Theory}

We have considered the click of a detector as the prototype of an event. It is a complex, macroscopic phenomenon. We may subdivide it and claim that it is initiated by an ionization process of an atom. But the boundaries of such subdivisions are not clear. We have no sharp definition of an elementary event. Of course, primary objective phenomena are necessarily coarse. But coarse phenomena are used to infer finer features. Take the example of electron-positron collisions in a storage ring. Here a detailed history of sequences of individual high energy events is constructed from traces of particles connecting them. The events in which we are interested are not the detector clicks. They constitute only a minor disturbance of the processes. We see a loc- alization of the vertices of significant events which is much sharper than the overlap of wave functions, the extension of a detector and presumably much sharper then the reconstruction from the traces reveals. And we cannot regard such an event as the measurement of some local observable. The refinement of the notion of event is a question of extrapolation (determining the limitations in the extension of objective appearance).

For this standard language and formalism of existing theory offers little help. Strictly speaking it says that no individual facts are created unless we take recourse to some faculties of human observers quite apart from consciousness: the will to pose a question to nature and the intelligence to construct appropriate measuring instruments. Schrödinger brought home this point with his paradox of the cat which, by its grotesque exaggeration, seems to show that not even coarse events have a place in the theory unless we regard the cat as a measuring instrument for the breaking of a bottle of poison and have a veterinarian at hand who can read the result. Here the discussions of decoherence due to macroscopic size or enviremental influence help to console us, telling us that the statistics over the fate of many cages of cats does not contradict the assumption that in each individual case the bottle of poison will be either broken or unbroken within some chosen, not too small, time interval, irrespective of how we try to verify this.

But the problem lies deeper. The asymmetric treatment of the emergence of facts as an interaction between a (large) measuring instrument with a (possibly small) system is pragmatically useful but not appropriate in a fundamental theory. Experimental equipment is part of objective appearance, irrespective of whether it is wilfully constructed and placed somewhere. The concept of an "observable" involving the choice by an experimenter to provide the scene for the emergence of facts is a deus ex machina with serious flaws. The very elaborate, precise mathematical structure in which self adjoint operators are supposed to represent observables contrasts strangely with the very unprecise description of extended objective appearance. In simple cases, essentially the quantum mechanics of a definite number of particles moving in external fields, the bridge relies on the correspondence with a pre-existing classical theory modified by formal rules of quantization. With its help we can guess self adjoint operators which correspond to simple dynamical variables of the classical theory. In relativistic theory we rely instead on a classification of observables in terms of space-time regions which provides an adequate bridge to the asymptotic analysis of 
collision processes. But again this involves some unsatisfactory idealizations. We can be pretty sure that most of the self adjoint operators have no counterpart in realizable equipment even if unlimited financial resources were available. The equipment used must comply with the laws of nature and not selfconsistent treatment of this problem is possible in the standard language.

There are the two tasks:

1) Passing from past objective appearance to the description of the relevant quantum state in which the probability assignments for future phenomena are encoded.

2) Classification of possible phenomena and description of the set of alternatives for their emergence.

Progress in the past decades has put us in a better position than the one faced in 1930. The correspondence principle has kept some heuristic value but it is not essential for the interpretation of the formalism. One basic ingredient for this is now the symmetry group of the theory. At present it consists of the Poincare' group, relating to global energy-momentum, angular momentum, center of mass position, and of the gauge groups relating to generalized charge structure (including lepton number, baryon number ...). This allows the characterization of simple global states such as vacuum, single particle states, thermodynamic equilibrium states to specified temperature and an array of chemical potentials. The division of space-time allows us to define "local observables", (roughly) representing detectors and coincidence arrangements of detectors. Thereby we can define "partial states" of chosen space-time regions. These, in turn, can be classified by considering simple global states whose restriction to the envisaged region approximates the situation prevailing there. For suitable divisions of space the mentioned simple global states suffice for an adequate description of the partial states prevailing initially in an experiment. It may be worthwhile to mention here an operational definition of the global states describing a single, stable particle. These are states which cannot produce a coincidence of any two detectors placed space-like to each other at any time. They are not localized at a given time because they can produce a click here or there but they are "simply localized" at all times because they can never produce two simultaneous clicks in separated detectors. If we disregard the infrared problems of Quantum Electrodynamics then this operational definition is equivalent to Wigner's mathematical definition of single particle states as rays in the space of an irreducible representa- tion of the symmetry group. This, in turn, is equivalent to a wave equation together with the relativistic generalization of Heisenberg's commutation relations.

Concerning the second task we are not in a satisfactory position. The theory divides the emergence of a fact into two stages. There is the choice by the observer, mathematically represented as the choice of a family of mutually orthogonal projectors. And there is the choice by nature in answering the posed multiple choice question in each individual case. I have criticized above this asymmetric treatment of the emergence of facts. But the remedy is not easy. It relates to other indications of deep lying problems. Thus the mentioned symmetry group can have only local significance. This is suggested by the local gauge principle which acquired a dominent role in high energy physics. And - for the space-time symmetry - it is suggested by General Relativity. This means that we have to consider the (Cartesian) product of many copies of the symmetry group and in the relation between them there enters the concept of connections. On the conceptual side this means that we should not regard space-time as a pregiven arena. For an operational definition of regions we need the concept of events. We know that global gauge invariance implies some modification of the superposition principle, so called superselection rules. We may expect that local gauge invariance leads to a more serious limitation of the superposition principle which is needed for a sharper definition of events. Compare the talk by Roger Penrose where limitations due to General Relativity were discussed. There are also indications that spatial and gauge symmetries should be unified and can only approximately be treated separately.

\section{The Cut and the Arrow of Time}

Let us now move to the next aspect. The content of objective appearance depends on time, or rather it increases with increasing time because we can find records of past phenomena but cannot see the future. This would have no fundamental significance for physics if physics dealt with a real universe existing externally and governed by deterministic laws. But if we attach reality only to the extended realm of objective appearance and if the theory is intrinsically limited to probability statements about the occurrence of phenomena then the arrow of time acquires a fundamental significance. We come to an evolutionary picture of reality in which we have an ever shifting cut between two realms: a past, 
constituting the realm of objective appearance for some group of observers (or, if you prefer, attributed to existing collective consciousness) and a future, the realm of possibilities, described by probability assignments for the way of growth of objective appearance. The boundary (the present) remains unsharp since we have no clear definition of elementary events, no sharp subdivision of coarse phenomena. Of course the "arrow" does not refer to clock-time but to the ordering of causal relations between events.

Let me finally compare what I have said with the standard language, in particular with the Copenhagen Interpretation and its center piece, the epistemological considerations of Niels Bohr. There we have a cut between the world of the observer - whose instruments must be described in the "language of classical physics" - and a physical system which is singled out for observation. In my picture we have a cut between past and future relative to some group of conservers. On the one side lies the extended objective appearance of the past, considered as factual. On the other probability assignments for future patterns of phenomena which are infered from past facts ${ }^{1}$.

Most physicists are reluctant to accord a fundamental role of the arrow of time in the theory. There is detailed balancing, time reversal invariance (or at least PCT-invariance) of the dynamical laws and we were educated in the credo that the second law of thermodynamics results from coarse graining of the description. But one has to be careful. In classical theory the coarse graining alone is not sufficient. On must add physically plausible assumptions, for instance that we start from an initial state with low thermodynamic probability and ask for its development in the future (not where it came from in the past). In Quantum Physics this assumption may be unnecessary. But then we must recognize that time re-

[1] R. Haag, Local Quantum Physics, $2^{\text {nd }}$ edition, SpringerVerlag, Berlin 1996.

[2] D. Buchholz and R. Haag, The quest for understanding in relativistic quantum physics, J. Math. Phys. Special Issue, June 2000 versal describes a symmetry within the realm of probability assignments whereas the irreversibility I refer to arises in the transition from possibilities to facts. If one ignores in the theory the emergence of individual facts, the primary experience from which the theory starts, one has only an empty formalism. In the standard language facts appear as results of observation and Niels Bohr mentions "the essential irreversibility inherent in the very concept of observation". It may be only a matter of words whether one speaks of the emergence of a fact or of the result of an observation. But we cannot limit this to an interaction process between a physical system and a measuring instrument wilfully placed somewhere by an experimenter. Both are part of objective appearance and the different treatment, while pragmatically useful, does not correspond to a fundamental difference.

The sketch I have drawn above depends on three ingredients: the interpretation of reality via objective appearance, its rough divisibility into elements called events and the acceptance of intrinsic indeterminacy. There are some murky areas, especially concerning the division of reality into individual events (which can be considered as facts), a problem intimately related to our understanding of space and time. We see indications heralding deep changes of the foundations of the theory. I do not want to venture here into speculations about the shape of future theory. It may change the assessment of all the mentioned ingredients. But I do not believe that any of the existing proposals for the elimination of intrinsic indeterminacy will be fruitful nor do I see that the "many world picture", devised to soften its impact, is of help. "Reality" at all times relates to common experience. It grows with time but does not split though there are alternative possibilities for its evolution.

[3] R. Haag, Questions in quantum physics, a personal view, in Mathematical Physics 2000; A. Fokas, A. Grygorian, T. Kibble, and B. Zegarlinski (ed) Imperial College Press, London.

\footnotetext{
${ }^{1}$ One might object that we can include "post selection criteria" in the evaluation of statistics. But the quantum theoretical rules for calculating probabilities are not symmetric with respect to an exchange of prior and post facts. Pre-selection cannot be omitted; it defines the quantum state. Post selection then restricts attention to a subensemble which, in general is not representable by a density matrix.
} 